\title{
Fractal two-level finite element method for free vibration of cracked beams
}

\author{
A.Y.T. Leung* \\ School of Engineering, University of Manchester, \\ Manchester M13 9PL, UK \\ R.K.L. Su \\ Ove Arup \& Partners, Hong Kong
}

\begin{abstract}
The fractal two-level finite element method is extended to the free vibration behavior of cracked beams for various end boundary conditions. A cracked beam is separated into its singular and regular regions. Within the singular region, infinite number of finite elements are virturally generated by fractal geometry to model the singular behavior of the crack tip. The corresponding numerous degrees of freedom are reduced to a small set of generalized displacements by fractal transformation technique. The solution time and computer storage can be remarkably reduced without sacrifying accuracy. The resonant frequencies and mode shapes computed compared well with the results from a commercial program.
\end{abstract}

\section{Introduction}

In many structures such as plates and beams, cracks may be developed as a results of corrosion or cyclic loading. It is well known that cracks in a structure may introduce considerable change in its natural frequencies and mode shapes. Dynamic analyses of cracked structures are important in order to detect cracks in the structures (Davies and Mayes [6] and $\mathrm{Hu}$ and Liang [5]). Several approaches have been used to analyse vibration of cracked structures. Examples are the line-spring model by Miyazaki [7], Dimarogonas' crack model [3] and infinitely small element method by Go and Lin [4]. In dynamic analysis, Davies and Mayes [2] have suggested that a relatively small crack can generally be regarded as a slot in the structure. Therefore it is often sufficient to neglect the crack closure effect for small crack depth ratios and it has

\footnotetext{
*Corresponding author: Tel.: +44 161275 4313; Fax: +44 161 275 4346; E-mail: Andrew.Leung@man.ac.uk.
}

been adopted by many researchers such as Dimarogonas and Papadopoulos [3] and Go and Lin [4]. It will be shown in this paper that both the resonant frequencies and the mode shapes of cracked beams are not sensitive to small crack-depth ratios. Therefore, crack detection methods based on change of modes may not work for small crack-depth ratios.

The fractal two-level finite element method developed by Leung and $\mathrm{Su}$ [9] (F2LFEM) will be extended to determine the resonant frequencies and mode shapes of two-dimensional cracked structures. In F2LFEM, we suggest that while the local interpolating shape functions within a finite element reduce the infinite number of degrees of freedom of a continuum to a finite number of degrees of freedom associated with the nodal displacements, global interpolating functions for the nodal displacements can also reduce the number of unknowns significantly. In order to avoid the troublesome mesh refinement around the crack tip and retain the advantage of agility of conventional finite element method, a cracked structure is separated into its singular (enclosing the crack) and regular regions. Within the singular region, infinite number of finite elements are virturally generated by fractal geometry to model the singular behavior of the crack tip. The corresponding numerous nodal displacements are reduced to a small set of generalized displacements by fractal transformation technique. The regular region is modeled by conventional finite elements. By this approach, the computer storage and the solution time for the eigenvalue problems can be effectively reduced. Accuracy in the stress intensities is increased as the round off errors are minimized with respect to the greatly reduced number of unknowns. Examples of cracked beams are given to demonstrate the efficiency of the present method. The results are in close agreement with those obtained from the commerical finite element package COSMOS/M but the present method requires only $10 \%$ of the computing memory. 


\section{Stiffness and mass matrices for similar 2D finite elements}

The conventional isoparametric finite element method (Zienkiewicz and Taylor [12]) suggests the following interpolation for the coordinates $\{x\}$ and the displacements $\{u\}$

$$
\begin{aligned}
& \{x\}=[N(\xi, \eta)]\{X\}, \\
& \{u\}=[N(\xi, \eta)]\{d\},
\end{aligned}
$$

where $[N(\xi, \eta)]$ are the shape functions in terms of natural coordinates $(\xi, \eta),\{X\}$ are the nodal coordinates and $\{d\}$ are the nodal displacements. By the variational method (Zienkiewicz and Taylor [12]), the dynamic equilibrium equation for free vibration of the assembled structure can be written symbolically as,

$$
\left[K-\omega^{2} M\right]\{\bar{D}\}=\{0\},
$$

in which $[K]$ is the global stiffness matrix, $[M]$ is the global mass matrix, $\{\bar{D}\}$ are the amplitudes of nodal displacements of vibration and $\omega$ is the natural frequency.

Considering two sets of elements denoted by 1 and 2 , which are similar in shape but the length dimensions are different in ratio $\alpha(=1 / 2$, say), the coordinates for the second set of elements can be expressed as $\left\{X_{i}^{2}\right\}=\alpha\left\{X_{i}^{1}\right\}$. Leung and Su [10] have shown that the stiffness matrices of elements set 1 are exactly similar to those of elements set 2 . Therefore we have

$$
\left[K^{2}\right]=\left[K^{1}\right]
$$

whereas the mass matrices for element 2 can be related with those for element 1 by the following relationship,

$$
\left[M^{2}\right]=\alpha^{2}\left[M^{1}\right] .
$$

Equations (3) and (4) will be used for similar substructures later.

\section{Global interpolation function}

Williams [11] used the eigenfunction method to derive the expressions of the displacements at the crack tip for $2 \mathrm{D}$ plane crack. The resulting displacement series are listed here:

$$
\begin{aligned}
u=\sum_{n=0}^{\infty} & \frac{r^{n / 2}}{2 \mu}\left\{a _ { 2 n + 1 } \left[\left(\kappa+\frac{n}{2}+(-1)^{n}\right)\right.\right. \\
& \left.\times \cos \frac{n}{2} \theta-\frac{n}{2} \cos \frac{n-4}{2} \theta\right] \\
& -a_{2 n+2}\left[\left(\kappa+\frac{n}{2}-(-1)^{n}\right)\right. \\
& \left.\left.\times \sin \frac{n}{2} \theta-\frac{n}{2} \sin \frac{n-4}{2} \theta\right]\right\}, \\
v= & \frac{r^{n / 2}}{2 \mu}\left\{a _ { 2 n + 1 } \left[\left(\kappa-\frac{n}{2}-(-1)^{n}\right)\right.\right. \\
& \left.\times \sin \frac{n}{2} \theta+\frac{n}{2} \sin \frac{n-4}{2} \theta\right] \\
+ & a_{2 n+2}\left[\left(\kappa-\frac{n}{2}+(-1)^{n}\right)\right. \\
& \left.\left.\times \cos \frac{n}{2} \theta+\frac{n}{2} \cos \frac{n-4}{2} \theta\right]\right\},
\end{aligned}
$$

in which $(r, \theta)$ is the system of polar coordinates originating form crack tip, $\mu$ is the shear modulus, $\nu$ is the Poisson's ratio and $\kappa$ is equal to $3-\nu$ for plane strain and $(3-\nu) /(1+\nu)$ for plane stress problems. The coefficients $a_{m}$ can be determined after imposing the loading and boundary conditions. It should be noted that $a_{1}$ and $a_{2}$ provide the necessary rigid body translations for the fractal mesh, and the terms $a_{3}$ and $a_{4}$ correspond to the singular stress terms representing the singular behavior at the crack tip.

\section{The fractal two-level finite element method}

Equation (5) shows that the displacements near the crack tip do not vary arbitrarily but follow certain displacement patterns which automatically satisfy the boundary conditions at crack surface. These displacement patterns are good global interpolating functions for F2LFEM to interpolate the nodal displacements near the crack tip. By this approach, the number of degrees of freedom is greatly reduced and computing efforts to solve the eigenvalue problem in Eq. (2) are remarkably simplified.

The nodal displacements $\{d\}=\left\{u_{i}\left(X_{i}, Y_{i}\right)\right.$, $\left.v_{i}\left(X_{i}, Y_{i}\right)\right\}=\left\{u_{i}\left(r_{i}, \theta_{i}\right), v_{i}\left(r_{i}, \theta_{i}\right)\right\}$ can be transformed by Eq. (5) to a set of generalized coordinates $\{a\}$ where $X_{i}, Y_{i}$, and $r_{i}, \theta_{i}$ are Cartesian and polar coordinates at node $i$, respectively. It can be represented by the equation 


$$
\{d\}=[T(r, \theta)]\{a\}
$$

where $[T]$ is the transformation matrix that can be determined by Eq. (5).

\section{Fractal transformation}

The concept of self-similarity in fractal geometry is employed to generate infinite layers of finite element meshes around the crack tip (Fig. 1). The infinite number of nodal displacements around the crack tip are transformed to a finite number (equal to 20 in the present case) of generalized displacements $\{a\}$ by using the fractal transformation technique. The technique can be explained by considering the transformation of the outermost layer of fractal mesh and the inner layers of fractal mesh sequentially.

For the outermost (first) layer of mesh, let the amplitudes of displacements on the boundary $\Gamma$ of the master nodes be $\left\{\bar{u}_{m}\right\}$ and those within the boundary $\Gamma$ of the slave nodes be $\left\{\bar{u}_{s}\right\}$. To carry out the transformation, the stiffness matrix and mass matrix in Eq. (2) are first partitioned with respect to $s$ and $m$, such that,

$$
\begin{aligned}
& {\left[\left[\begin{array}{cc}
K_{s s}^{\mathrm{f}} & K_{s m}^{\mathrm{f}} \\
K_{m s}^{\mathrm{f}} & K_{m m}^{\mathrm{f}}
\end{array}\right]\right.} \\
& \left.+\omega^{2}\left[\begin{array}{cc}
M_{s s}^{\mathrm{f}} & M_{s m}^{\mathrm{f}} \\
M_{m s}^{\mathrm{f}} & M_{m m}^{\mathrm{f}}
\end{array}\right]\right]\left\{\begin{array}{l}
\bar{u}_{s} \\
\bar{u}_{m}
\end{array}\right\}=\left\{\begin{array}{l}
0 \\
0
\end{array}\right\},
\end{aligned}
$$

where the superscript ' $f$ ' denotes the first layer of fractal mesh.

Since only the displacements at the slave nodes are transformed, second level (global) interpolation of displacements are written as,

$$
\left\{\begin{array}{c}
\bar{u}_{s} \\
\bar{u}_{m}
\end{array}\right\}=\left\{\begin{array}{ll}
T & 0 \\
0 & I
\end{array}\right\}\left\{\begin{array}{c}
a \\
\bar{u}_{m}
\end{array}\right\}
$$

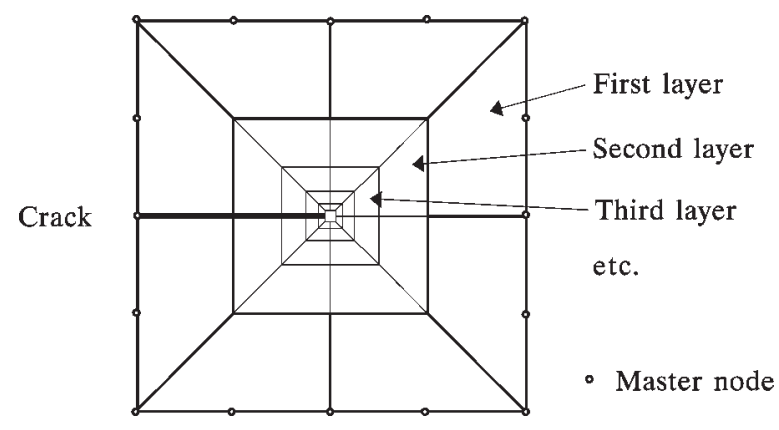

Fig. 1. Fractal mesh configuration. in which $[I]$ is the identity matrix. After performing the transformation, we have

$$
\begin{aligned}
& {\left[\left[\begin{array}{cc}
T^{\mathrm{T}} K_{s s}^{\mathrm{f}} T & T^{\mathrm{T}} K_{s m}^{\mathrm{f}} \\
K_{m s}^{\mathrm{f}} T & K_{m m}^{\mathrm{f}}
\end{array}\right]\right.} \\
& \left.+\omega^{2}\left[\begin{array}{cc}
T^{\mathrm{T}} M_{s s}^{\mathrm{f}} T & T^{\mathrm{T}} M_{s m}^{\mathrm{f}} \\
M_{m s}^{\mathrm{f}} T & M_{m m}^{\mathrm{f}}
\end{array}\right]\right]\left\{\begin{array}{c}
a \\
\bar{u}_{m}
\end{array}\right\}=\left\{\begin{array}{l}
0 \\
0
\end{array}\right\} .
\end{aligned}
$$

For the inner layers of fractal mesh, each element stiffness matrix within the first layer of $D$ would be transformed and assembled to form the generalized stiffness matrix. Considering the transformation of the $l$-th layer of inner elements, the transformation matrix of the $l$-th layer of mesh can be related with the first layer by,

$$
\left\{T_{j}^{l}(r, \theta)\right\}=\alpha^{(n-1)(l-1) / 2}\left\{T_{j}^{\mathrm{f}}(r, \theta)\right\}
$$

and

$$
n= \begin{cases}(j-1) / 2 & \text { for } j=1,3,5 \ldots, \\ (j-2) / 2 & \text { for } j=2,4,6 \ldots\end{cases}
$$

where $\left\{T_{j}^{l}\right\}$ and $\left\{T_{j}^{\mathrm{f}}\right\}$ are the column vectors of transformation matrices $\left[T^{l}\right]$ and $\left[T^{\mathrm{f}}\right]$, respectively. Any element $(i, j)$ in the generalized stiffness matrix and the generalized mass matrix can be written as,

$$
k_{i j}^{l}=\left\{T_{i}^{l}\right\}^{\mathrm{T}}\left[K^{l}\right]\left\{T_{j}^{l}\right\}
$$

and

$$
m_{i j}^{l}=\left\{T_{i}^{l}\right\}^{\mathrm{T}}\left[M^{l}\right]\left\{T_{j}^{l}\right\} .
$$

By substituting Eqs (3), (4) and (10) into Eqs (12) and (13), we get

$$
k_{i j}^{l}=\alpha^{(n+m-2)(l-1) / 2}\left\{T_{i}^{\mathrm{f}}\right\}^{\mathrm{T}}\left[K^{\mathrm{f}}\right]\left\{T_{j}^{\mathrm{f}}\right\}
$$

and

$$
m_{i j}^{l}=\alpha^{(n+m+2)(l-1) / 2}\left\{T_{i}^{\mathrm{f}}\right\}^{\mathrm{T}}\left[M^{\mathrm{f}}\right]\left\{T_{j}^{\mathrm{f}}\right\},
$$

where

$$
m= \begin{cases}(i-1) / 2 & \text { for } i=1,3,5 \ldots, \\ (i-2) / 2 & \text { for } i=2,4,6 \ldots\end{cases}
$$

Finally, to determine the generalized global stiffness and mass matrices, we super-impose $k_{i j}^{l}$ and $m_{i j}^{l}$ from layer 2 to the infinite layer. It is a geometrical progression series and subsequently the transformation of infinite number of elements at the inner layers can be readily obtained,

$$
\sum_{l=2}^{\infty} k_{i j}^{l}=\frac{R_{n m}}{1-R_{n m}} k_{i j}^{\mathrm{f}}
$$

and 


$$
\sum_{l=2}^{\infty} m_{i j}^{l}=\frac{S_{n m}}{1-S_{n m}} m_{i j}^{\mathrm{f}}
$$

in which $R_{n m}=\alpha^{(n+m-2) / 2}$ and $S_{n m}=\alpha^{(n+m+2) / 2}$. Using the Eqs (7), (17) and (18) the global generalized stiffness and mass matrices can be effectively evaluated. The resulting eigenvalue and eigenvector problems can be solved efficiently by subspaces iteration (Cheung and Leung [1]).

\section{Numerical examples}

A cracked beam which has dimensions $0.2 \mathrm{~m} \times$ $0.2 \mathrm{~m} \times 3 \mathrm{~m}$ is shown in Fig. 2. The material properties are Young's modulus $E=208.6 \mathrm{GPa}$, mass density $\rho=7.833 \mathrm{Mg} / \mathrm{m}^{3}$ and Poisson's ratio $\nu=0.3$. A crack is located at $L / 3$ from the left end of beam and the relative crack depth $a / h$ is ranged from 0.1 to 0.6 in which $a$ is the crack length and $h$ is the depth of beam. Different displacement boundary conditions are studied. In the sequel the letters S, C and F stand for simply, clamped and freely supported conditions, respectively. For example SC will mean a beam simply-supported at the left side and clamped at the right side.

The mesh configurations for crack depth ratio of $a / h=0.5$ are shown in Fig. 3. The 9-node Lagrangian elements are employed to model the cracked beam. The results of resonant frequencies of the beam for varying crack depth are listed in Table 1 . The results of the present study are compared with those from the finite element computer program COSMOS/M. Present analysis uses about 70 finite ele-

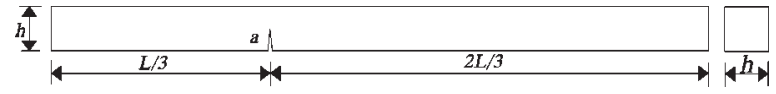

Fig. 2. Configuration of cracked beam. $L=3.0 \mathrm{~m}, h=0.2 \mathrm{~m}$, $E=208.6 \mathrm{GPa}$, density $=7.833 \mathrm{Mg} / \mathrm{m}^{3}$, Poisson's ratio $=0.3$.

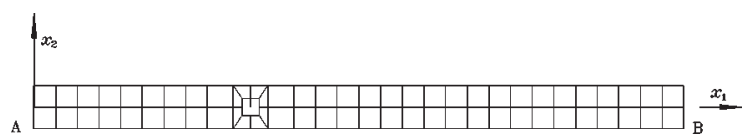

(a)

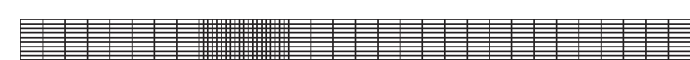

(b)

Fig. 3. Finite element meshes of cracked beam. F2LFEM mesh (a) and COSMOS/M - mesh (b).

ments as opposed to 640 in COSMOS/M. The results appear to be in close agreement. The resonant frequencies obtained by the COSMOS/M are in general smaller. It may be due to the fact that COSMOS/M use the reduced integration scheme (Zienkiewicz and Taylor [12]) while the present analysis apply full integration scheme. According to Table 1, for crack depth ratios $a / h=0.2$ and 0.6 , the reduction of resonant frequencies is usually less than $2.0 \%$ and $20.0 \%$, respectively. This implies that the resonant frequencies are not sensitive to the small crack-depth ratios. It can be explained by the fact that the resonant frequencies are the global parameters while cracking is a local effect. Small cracks $(a / h<0.2)$ affect only the local stress and displacement distributions of vibration beams. And, it has only a small variation in the inertia energy and kinetic energy.

Table 1

Resonant frequencies for cracked beams.

(a) Resonant frequencies for CC cracked beams

\begin{tabular}{lllllllll}
\hline$a / h$ & Mode 1 & Mode 2 & Mode 3 & Mode 4 & Mode 5 & Mode 6 & Mode 7 & Mode 8 \\
\hline 0.0 & 721.1 & 1919.1 & 3604.0 & 5413.5 & 5673.9 & 8045.2 & 10649.0 & 10821.6 \\
& $(0.04)$ & $(0.10)$ & $(0.05)$ & $(0.01)$ & $(0.16)$ & $(0.27)$ & $(0.25)$ & $(0.03)$ \\
0.1 & 720.3 & 1910.6 & 3603.6 & 5412.2 & 5659.2 & 8012.7 & 10650.0 & 10820.3 \\
& $(0.06)$ & $(0.09)$ & $(0.06)$ & $(0.05)$ & $(0.16)$ & $(0.27)$ & $(0.27)$ & $(0.07)$ \\
0.2 & 717.8 & 1887.9 & 3601.4 & 5402.7 & 5623.0 & 7930.0 & 10641.7 & 10815.3 \\
& $(0.20)$ & $(0.24)$ & $(0.07)$ & $(0.16)$ & $(0.27)$ & $(0.41)$ & $(0.26)$ & $(0.22)$ \\
0.3 & 714.0 & 1852.5 & 3596.2 & 5359.2 & 5588.3 & 7818.5 & 10610.9 & 10801.8 \\
& $(0.45)$ & $(0.46)$ & $(0.05)$ & $(0.22)$ & $(0.53)$ & $(0.64)$ & $(0.14)$ & $(0.46)$ \\
0.4 & 704.3 & 1797.8 & 3589.8 & 5252.0 & 5545.1 & 7657.2 & 10578.2 & 10720.6 \\
& $(0.19)$ & $(0.22)$ & $(0.06)$ & $(0.09)$ & $(0.31)$ & $(0.39)$ & $(0.19)$ & $(0.21)$ \\
0.5 & 694.6 & 1741.7 & 3580.7 & 5103.8 & 5532.6 & 7523.2 & 10497.5 & 10656.9 \\
& $(0.19)$ & $(0.21)$ & $(0.05)$ & $(0.06)$ & $(0.29)$ & $(0.37)$ & $(0.16)$ & $(0.19)$ \\
0.6 & 684.3 & 1689.1 & 3569.7 & 4937.8 & 5527.6 & 7417.6 & 10368.3 & 10587.3 \\
& $(0.23)$ & $(0.29)$ & $(0.05)$ & $(0.09)$ & $(0.28)$ & $(0.40)$ & $(0.13)$ & $(0.16)$ \\
\hline
\end{tabular}

The percentage difference of present results with COSMOS/M results are shown within brackets. 
Table 1

(Continued)

(b) Resonant frequencies for CS cracked beams.

\begin{tabular}{lllllllll}
\hline$a / h$ & Mode 1 & Mode 2 & Mode 3 & Mode 4 & Mode 5 & Mode 6 & Mode 7 & Mode 8 \\
\hline 0.0 & 501.7 & 1576.4 & 2704.5 & 3153.6 & 5128.4 & 7400.5 & 8110.8 & 9882.1 \\
& $(0.02)$ & $(0.10)$ & $(0.00)$ & $(0.10)$ & $(0.17)$ & $(0.36)$ & $(0.03)$ & $(0.38)$ \\
0.1 & 501.6 & 1569.1 & 2699.6 & 3150.7 & 5121.4 & 7367.1 & 8116.2 & 9874.8 \\
& $(0.05)$ & $(0.10)$ & $(-0.02)$ & $(0.10)$ & $(0.17)$ & $(0.36)$ & $(0.09)$ & $(0.40)$ \\
0.2 & 501.1 & 1548.8 & 2682.2 & 3142.8 & 5101.0 & 7281.4 & 8132.4 & 9850.0 \\
& $(0.14)$ & $(0.26)$ & $(-0.02)$ & $(0.14)$ & $(0.22)$ & $(0.51)$ & $(0.29)$ & $(0.44)$ \\
0.3 & 500.5 & 1514.3 & 2638.6 & 3132.2 & 5067.9 & 7163.7 & 8167.9 & 9805.7 \\
& $(0.31)$ & $(0.46)$ & $(-0.34)$ & $(0.20)$ & $(0.26)$ & $(0.79)$ & $(0.73)$ & $(0.41)$ \\
0.4 & 497.3 & 1456.6 & 2582.9 & 3118.0 & 5020.9 & 6987.9 & 8132.5 & 9751.2 \\
& $(0.13)$ & $(0.18)$ & $(-0.16)$ & $(0.15)$ & $(0.21)$ & $(0.51)$ & $(0.29)$ & $0.40)$ \\
0.5 & 493.8 & 1384.4 & 2492.8 & 3108.2 & 4972.1 & 6837.1 & 8132.5 & 9686.3 \\
& $(0.12)$ & $(0.08)$ & $(-0.28)$ & $(0.16)$ & $(0.22)$ & $(0.50)$ & $(0.29)$ & $(0.38)$ \\
0.6 & 489.1 & 1295.2 & 2379.9 & 3102.0 & 4928.3 & 6716.2 & 8132.5 & 9613.0 \\
& $(0.12)$ & $(0.04)$ & $(-0.35)$ & $(0.17)$ & $(0.25)$ & $(0.54)$ & $(0.29)$ & $0.37)$ \\
\hline
\end{tabular}

The percentage difference of present results with COSMOS/M results are shown within brackets.

(c) Resonant frequencies for CF cracked beams.

\begin{tabular}{lllllllll}
\hline$a / h$ & Mode 1 & Mode 2 & Mode 3 & Mode 4 & Mode 5 & Mode 6 & Mode 7 & Mode 8 \\
\hline 0.0 & 116.1 & 713.4 & 1938.4 & 2704.5 & 3649.3 & 5758.6 & 8110.8 & 8178.5 \\
& $(0.02)$ & $(0.08)$ & $(0.17)$ & $(0.00)$ & $(0.14)$ & $(0.28)$ & $(0.03)$ & $(0.39)$ \\
0.1 & 115.7 & 712.0 & 1930.0 & 2699.7 & 3649.0 & 5742.0 & 8116.1 & 8146.6 \\
& $(-0.04)$ & $(0.10)$ & $(0.16)$ & $(-0.01)$ & $(0.16)$ & $(0.45)$ & $(0.09)$ & $(0.40)$ \\
0.2 & 114.3 & 708.2 & 1906.7 & 2683.3 & 3647.3 & 5697.0 & 8065.0 & 8132.4 \\
& $(-0.10)$ & $(0.21)$ & $(0.31)$ & $(-0.01)$ & $(0.17)$ & $(0.37)$ & $(0.54)$ & $(0.29)$ \\
0.3 & 111.7 & 701.6 & 1866.8 & 2645.0 & 3642.8 & 5629.3 & 7954.2 & 8167.9 \\
& $(-0.41)$ & $(0.38)$ & $(0.47)$ & $(-0.27)$ & $(0.14)$ & $(0.53)$ & $(0.76)$ & $0.73)$ \\
0.4 & 108.4 & 688.4 & 1800.9 & 2599.7 & 3638.3 & 5527.1 & 7793.3 & 8132.5 \\
& $(-0.25)$ & $(0.17)$ & $(0.22)$ & $(-0.12)$ & $(0.15)$ & $(0.39)$ & $(0.52)$ & $(0.29)$ \\
0.5 & 102.9 & 671.6 & 1717.8 & 2530.6 & 3632.0 & 5429.8 & 7655.7 & 8132.5 \\
& $(-0.42)$ & $(0.13)$ & $(0.10)$ & $(-0.19)$ & $(0.15)$ & $(0.39)$ & $(0.49)$ & $(0.29)$ \\
0.6 & 94.7 & 649.1 & 1615.8 & 2447.9 & 3624.7 & 5348.2 & 7541.9 & 8132.5 \\
& $(-0.61)$ & $(0.12)$ & $(0.03)$ & $(-0.21)$ & $(0.15)$ & $(0.45)$ & $(0.51)$ & $(0.29)$ \\
\hline
\end{tabular}

The percentage difference of present results with COSMOS/M results are shown within brackets.

(d) Resonant frequencies for SC cracked beams.

\begin{tabular}{lllllllll}
\hline$a / h$ & Mode 1 & Mode 2 & Mode 3 & Mode 4 & Mode 5 & Mode 6 & Mode 7 & Mode 8 \\
\hline 0.0 & 501.7 & 1576.4 & 2704.5 & 3153.6 & 5128.4 & 7400.5 & 8110.8 & 9882.1 \\
& $(0.04)$ & $(0.07)$ & $(0.00)$ & $(0.08)$ & $(0.26)$ & $(0.28)$ & $(0.00)$ & $(0.37)$ \\
0.1 & 499.3 & 1571.9 & 2704.0 & 3152.6 & 5104.5 & 7386.8 & 8090.3 & 9878.0 \\
& $(0.02)$ & $(0.05)$ & $(0.04)$ & $(0.09)$ & $(0.25)$ & $(0.29)$ & $(-0.05)$ & $(0.40)$ \\
0.2 & 492.4 & 1559.4 & 2701.7 & 3149.1 & 5040.2 & 7344.3 & 8026.5 & 9861.7 \\
& $(0.14)$ & $(0.11)$ & $(0.18)$ & $(0.10)$ & $(0.40)$ & $(0.33)$ & $(-0.09)$ & $(0.44)$ \\
0.3 & 480.0 & 1538.0 & 2693.9 & 3142.0 & 4939.2 & 7257.3 & 7901.3 & 9831.7 \\
& $(0.23)$ & $(0.13)$ & $(0.35)$ & $(0.08)$ & $(0.62)$ & $(0.24)$ & $(-0.42)$ & $(0.42)$ \\
0.4 & 459.8 & 1506.7 & 2664.1 & 3133.7 & 4782.1 & 7124.5 & 7807.9 & 9798.9 \\
& $(0.01)$ & $(0.06)$ & $(0.11)$ & $(0.09)$ & $(0.39)$ & $(0.14)$ & $(-0.07)$ & $(0.43)$ \\
0.5 & 432.2 & 1467.3 & 2622.7 & 3123.0 & 4612.7 & 6936.9 & 7694.1 & 9762.1 \\
& $(-0.14)$ & $(0.01)$ & $(0.04)$ & $(0.09)$ & $(0.35)$ & $(-0.03)$ & $(0.00)$ & $(0.42)$ \\
0.6 & 395.0 & 1420.4 & 2559.0 & 3111.8 & 4443.8 & 6715.0 & 7592.9 & 9721.4 \\
& $(-0.24)$ & $(0.01)$ & $(-0.04)$ & $(0.10)$ & $(0.39)$ & $(-0.14)$ & $(0.09)$ & $(0.41)$ \\
\hline
\end{tabular}

The percentage difference of present results with COSMOS/M results are shown within brackets. 
Table 1

(Continued)

(e) Resonant frequencies for SS cracked beams.

\begin{tabular}{lllllllll}
\hline$a / h$ & Mode 1 & Mode 2 & Mode 3 & Mode 4 & Mode 5 & Mode 6 & Mode 7 & Mode 8 \\
\hline 0.0 & 323.8 & 1262.0 & 2728.0 & 4609.8 & 4647.9 & 5403.2 & 6793.4 & 9175.3 \\
& $(0.02)$ & $(0.08)$ & $(0.09)$ & $(1.34)$ & $(1.08)$ & $(0.00)$ & $(0.37)$ & $(0.44)$ \\
0.1 & 322.3 & 1256.6 & 2728.2 & 4589.5 & 4644.8 & 5393.7 & 6772.7 & 9177.0 \\
& $(-0.01)$ & $(0.06)$ & $(0.10)$ & $(1.07)$ & $(1.34)$ & $(-0.01)$ & $(0.38)$ & $(0.46)$ \\
0.2 & 318.1 & 1241.8 & 2728.1 & 4519.2 & 4643.9 & 5363.5 & 6723.0 & 9171.9 \\
& $(0.07)$ & $(0.14)$ & $(0.10)$ & $(0.86)$ & $(1.66)$ & $(-0.01)$ & $(0.49)$ & $(0.46)$ \\
0.3 & 310.3 & 1216.5 & 2726.5 & 4372.3 & 4646.9 & 5303.1 & 6667.8 & 9160.4 \\
& $(0.03)$ & $(0.19)$ & $(0.06)$ & $(0.62)$ & $(1.77)$ & $(-0.17)$ & $(0.71)$ & $(0.36)$ \\
0.4 & 298.1 & 1179.1 & 2726.0 & 4160.4 & 4637.6 & 5249.4 & 6593.1 & 9153.6 \\
& $(-0.09)$ & $(0.06)$ & $(0.08)$ & $(0.57)$ & $(1.58)$ & $(0.04)$ & $(0.51)$ & $0.42)$ \\
0.5 & 279.9 & 1132.4 & 2723.6 & 3871.7 & 4631.6 & 5188.8 & 6552.2 & 9130.8 \\
& $(-0.27)$ & $(-0.01)$ & $(0.08)$ & $(0.30)$ & $(1.48)$ & $(0.14)$ & $(0.52)$ & $(0.40)$ \\
0.6 & 253.6 & 1077.4 & 2719.9 & 3542.9 & 4621.0 & 5136.5 & 6531.7 & 9095.4 \\
& $(-0.45)$ & $(-0.02)$ & $(0.07)$ & $(0.11)$ & $(1.37)$ & $(0.29)$ & $(0.55)$ & $(0.39)$ \\
\hline
\end{tabular}

The percentage difference of present results with COSMOS/M results are shown within brackets.

(f) Resonant frequencies for FC cracked beams.

\begin{tabular}{lllllllll}
\hline$a / h$ & Mode 1 & Mode 2 & Mode 3 & Mode 4 & Mode 5 & Mode 6 & Mode 7 & Mode 8 \\
\hline 0.0 & 116.1 & 713.4 & 1938.4 & 2704.5 & 3649.3 & 5758.6 & 8110.8 & 8178.5 \\
& $(0.02)$ & $(0.07)$ & $(0.17)$ & $(0.00)$ & $(0.16)$ & $(0.27)$ & $(0.00)$ & $(0.41)$ \\
0.1 & 116.1 & 710.3 & 1926.6 & 2704.1 & 3647.7 & 5746.5 & 8077.2 & 8157.0 \\
& $(0.05)$ & $(0.03)$ & $(0.12)$ & $(0.04)$ & $(0.16)$ & $(0.28)$ & $(0.01)$ & $(0.35)$ \\
0.2 & 116.1 & 701.3 & 1894.0 & 2702.2 & 3642.6 & 5711.7 & 7925.1 & 8154.0 \\
& $(0.17)$ & $(0.03)$ & $(0.20)$ & $(0.18)$ & $(0.17)$ & $(0.37)$ & $(0.10)$ & $(0.35)$ \\
0.3 & 116.2 & 684.3 & 1839.0 & 2697.0 & 3632.5 & 5651.2 & 7646.9 & 8168.3 \\
& $(0.39)$ & $(-0.13)$ & $(0.19)$ & $(0.38)$ & $(0.13)$ & $(0.48)$ & $(-0.26)$ & $(0.53)$ \\
0.4 & 115.5 & 659.9 & 1762.8 & 2673.3 & 3621.3 & 5549.6 & 7358.1 & 8150.9 \\
& $(0.15)$ & $(-0.10)$ & $(0.10)$ & $(0.14)$ & $(0.14)$ & $(0.38)$ & $(-0.05)$ & $(0.35)$ \\
0.5 & 114.9 & 621.5 & 1668.6 & 2646.9 & 3606.6 & 5419.7 & 7030.7 & 8145.3 \\
& $(0.12)$ & $(-0.26)$ & $(-0.10)$ & $(0.10)$ & $(0.12)$ & $(0.36)$ & $(-0.16)$ & $(0.36)$ \\
0.6 & 113.6 & 565.4 & 1563.2 & 2610.4 & 3590.4 & 5262.0 & 6732.6 & 8131.7 \\
& $(0.08)$ & $(-0.40)$ & $(-0.02)$ & $(0.07)$ & $(0.12)$ & $(0.37)$ & $(-0.20)$ & $(0.39)$ \\
\hline
\end{tabular}

The percentage difference of present results with COSMOS/M results are shown within brackets.

Table 2

Some computational information for the present method and COSMOS/M.

\begin{tabular}{lcc}
\hline & Present & COSMOS/M \\
\hline Total number of degrees of freedom & 679 & 2985 \\
Total length of global stiffness matrix & 17157 & 168156 \\
Maximum half-bandwidth & 122 & 70 \\
Average half-bandwidth & 25 & 56 \\
Total solution time, sec & 170 & 420 \\
Time for subspace iteration, sec & 160 & 281 \\
\hline
\end{tabular}

The meshes are shown in Fig. 3.

Some of the information about the computing time, degrees of freedom and required storage are tabulated in Table 2. It is observed that the computing time and memory required for the present method are only about $2 / 5$ and $1 / 10$ of those for COSMOS/M, respectively.

The mode shapes for selected displacement boundary conditions CF and SS are plotted in Figs 4 and 5, respectively. It is found that all the vibration modes of the cracked beams with small cracks $(a / h<0.2)$ are very close to those of corresponding perfect beams. This suggests that the mode shapes of the perfect beams can be a good approximation for the small cracked beams. Moreover, using this approximation for the mode shapes of $\mathrm{CC}$ beam, the vibration problems for large scale cracked frame can be studied by using dynamic substructuring method (Leung [8,9]). Finally, it is observed that the "peak" of the vibration modes which is nearest to the crack shift toward the crack with increasing crack-depth ratio. This effect is understandable when considering the vibration behavior of two beam segments connected in series by a hinge and a spring.

In all examples, we used 20 generalised displacements. In fact, the results are not sensitive to the number of generalised displacements taken as shown in Leung and $\mathrm{Su}$ [10]. 

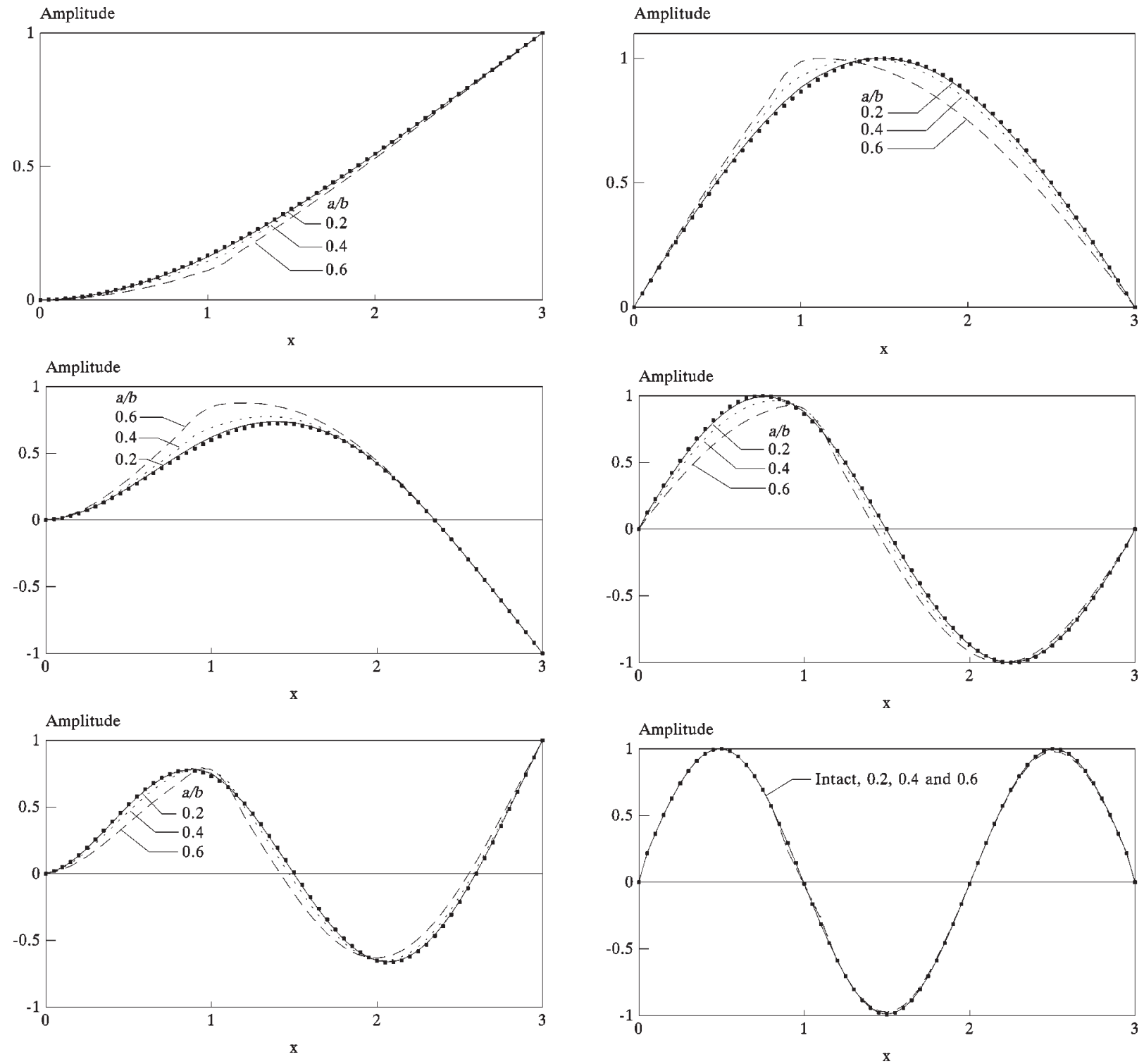

Fig. 4. Mode shapes for CF cracked beams (lines - cracked beams and dots - intact beam). (a) - 1st mode, (b) - 2nd mode, (c) 3rd mode.

\section{Conclusion}

The F2LFEM is extended to study the free vibration behavior of the cracked beams. Resonant frequencies and mode shapes for beams with various crack depth ratios and displacement boundaries are determined. The present results compare well with those obtained by COSMOS/M. The computing memory and solution time for solving the eigenvalue problems are remarkably reduced by the fractal transformation technique. It is found that both the reso-

Fig. 5. Mode shapes for SS cracked beams (lines - cracked beams and dots - intact beam). (a) - 1st mode, (b) - 2nd mode, (c) 3rd mode.

nant frequencies and mode shapes are not sensitive to small crack-depth ratios $(a / h<0.2)$. Therefore frequency monitoring by itself is not sufficient to detect a small crack initiating along a beams. Finally, the present method can be directly applied to analyze free vibration of multi-cracked beams and other twodimensional cracks problems without any difficulty. By combining with dynamic substructuring method, the vibration problem for large scale cracked frame can also be addressed. 


\section{References}

[1] Y.K. Cheung and A.Y.T. Leung, Finite Elements Methods in Dynamics, Kluwer, 1991.

[2] W.G.R. Davies and I.W. Mayes, The vibrational behavior of a multi-shaft, multi-bearing system in the presence of a propagating transverse crack, ASME Journal of Vibration, Acoustics, Stress a Reliability in Design 106(1) (1984), 146153.

[3] A.D. Dimarogonas and C.A. Papadopoulos, Vibration of cracked shafts in bending, Journal of Sound and Vibration, 91(4) (1983), 583-593.

[4] C.G. Go and Y.S. Lin, Infinitely small element for the dynamic problem of a cracked beam, Engineering Fracture Mechanics 48(4) (1994), 475-482.

[5] J. Hu and R.Y. Liang, An integrated approach to detection of cracks using vibration characteristics, Journal of the Franklin Institue 330(5) (1993), 841-853.
[6] I.W. Mayes and W.G.R. Davies, Analysis of response of a multi-rotor-bearing system containing a transverse crack in a rotor, ASME Journal of Vibrations, Acoustics, Stress and Reliability in Design 106(1) (1984), 139-145.

[7] N. Miyazaki, Application of line-spring model to dynamic stress intensity factor analysis of pre-cracked bending specimen, Engineering Fracture Mechanics 138 (1991), 321-326.

[8] Y.T. Leung, An accurate method of dynamic substructuring with simplified computation, International Journal for $\mathrm{Nu}$ merical Methods in Engineering 14 (1979), 1241-1256.

[9] A.Y.T. Leung, Dynamic Stiffness and Substructures, Springer, 1993.

[10] A.Y.T. Leung and R.K.L. Su, Mode I crack problems by fractal two level finite element methods, Engineering Fracture Mechanics 48(6) (1994), 847-856.

[11] M.L. Williams, On the stress distribution at the base of a stationary crack, Journal of Applied Mechanics 24 (1957), 109-114.

[12] O.C. Zienkiewicz and R.L. Taylor, The Finite Element Method, Vol. 1, McGraw-Hill, London, 1989.

Received 10 July 1995; Revised 13 February 1998 

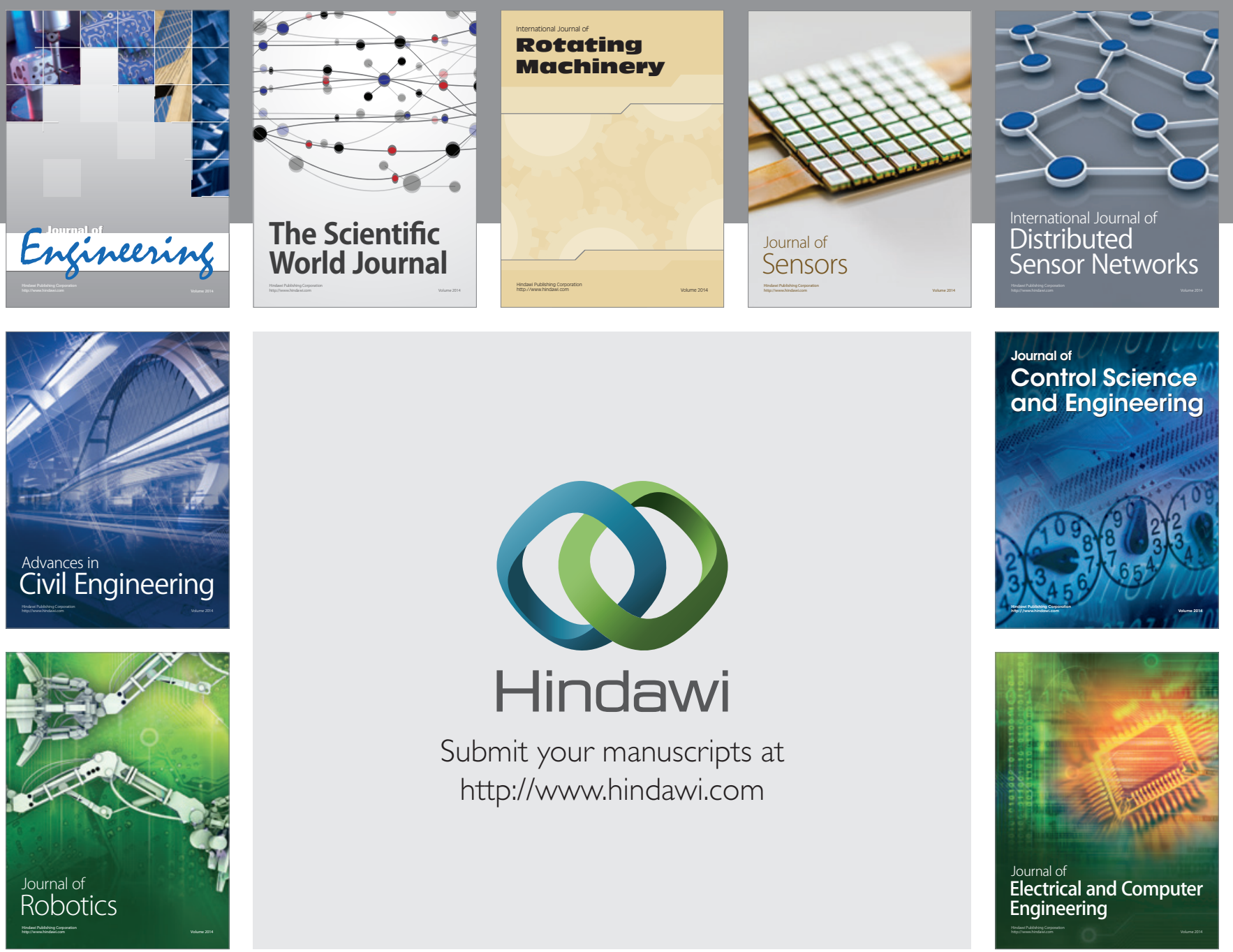

Submit your manuscripts at

http://www.hindawi.com
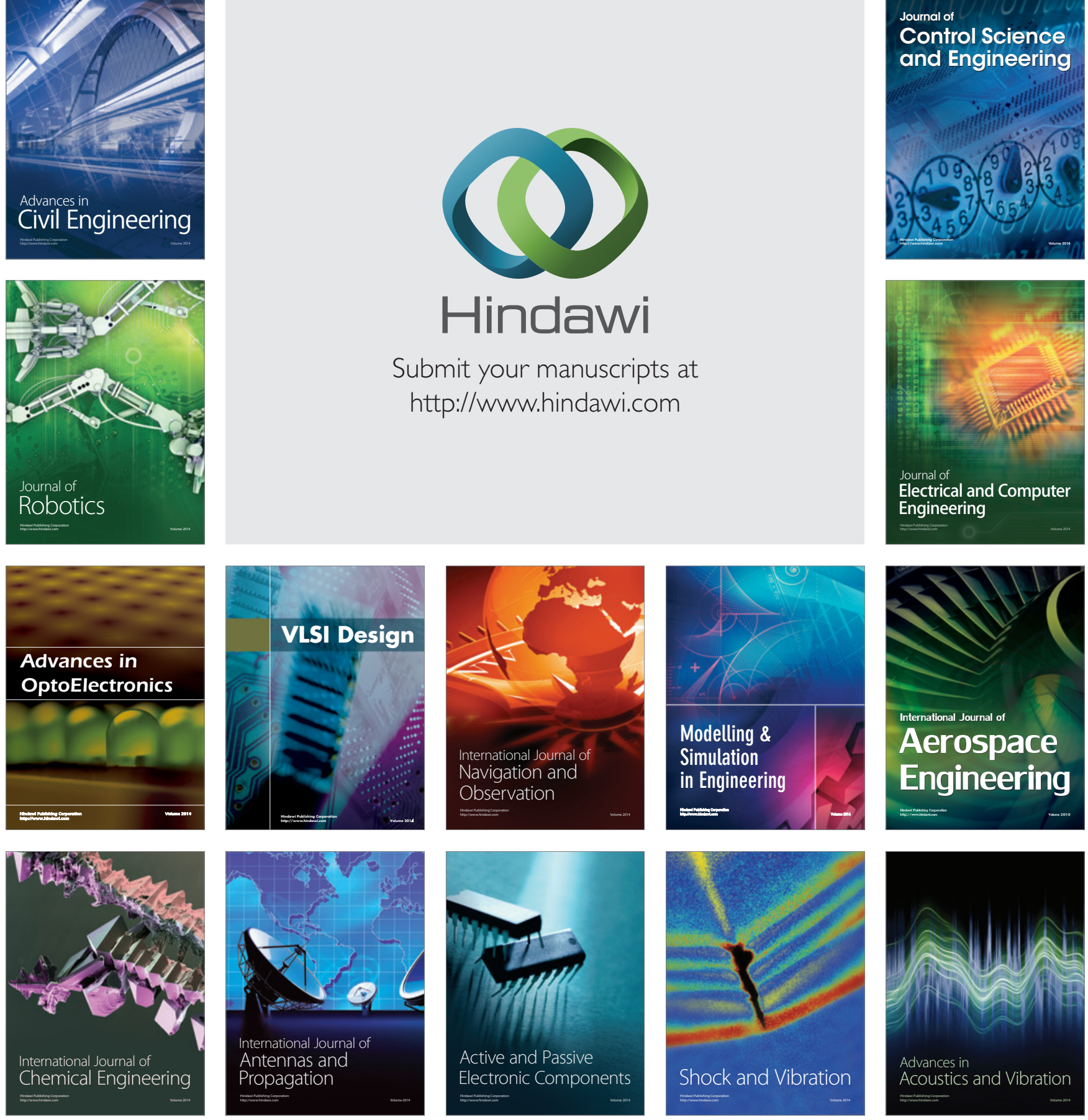